\title{
Entropy Maximization Problem in Network using Dijkstra's-Floyd Warshall Algorithm
}

\author{
Pawan Kumar Gupta \\ Assistant Professor \\ Department of Information Technology \\ AITR, Indore
}

\begin{abstract}
The shortest path problem is to find a path between two vertices on a given graph, such that the sum of the weights on its constituent edges is minimized. The classic Dijkstra's algorithm was designed to solve the single source shortest path problem for a static graph. It works starting from the source node and calculating the shortest path on the whole network. This work aims to develop a Hybrid algorithm Dijkstra's - Floyd Warshall algorithm to solve entropy maximization routing protocol problem. The algorithm has to find the shortest path between the source and destination nodes. Route guidance algorithm is use to find best shortest path in routing network, this is poised to minimize costs between the origin and destination nodes. The proposed algorithm is compared with the existing in order to find the best and shortest paths.
\end{abstract}

\section{General Terms}

Dijkstra's Algorithm, Floyd-Warshall Algorithm, Hybrid algorithm Dijkstra's-Floyd Warshall (HDFWA), Entropy.

\section{Keywords}

Entropy; Dijkstra's Algorithm; Dijkstra's-Floyd Warshall algorithm

\section{INTRODUCTION}

In calculation, the network usually refers to a mathematical graph. The system is composed of nodes, arcs and curve masses. The network stands called traditional if arc heaviness is coefficients. In recent ages network optimization consumes become a significant research gratified in working Research. It comprises areas such as straight path, network flows, and carriage routing then Chinese postman difficulties and so decided. The straight path problem distillates on finding a track with smallest distance, period, or cost after the source bulge to the terminus node. Some descriptive algorithms stayed proposed through Bellman, Dijkstra's, Dreyfus, in addition Floyd, which complete the conservative path problematic, occupy a dominant position in a system.

\section{Entropy}

In Data Communication, Entropy is Relative level of Randomness. Entropy is specifically relative to the greatest sensible information speed in bps. When we increment the entropy then it additionally expanded the many-sided quality of flag mistake. It is likewise specifically corresponding to commotion and data transfer capacity. The entropy in a flag is contrarily relative to compressibility; the more noteworthy the entropy, the littler the factor by which the information can be packed. Entropy additionally alludes to clutter purposely added to information in certain encryption forms.

\section{Principle of Maximum Entropy}

The Principle of Maximum Entropy expresses that, the likelihood dissemination which best speaks to the present condition of information is the one with biggest entropy.

\begin{abstract}
Network
A connection between two or more peripheral devices that is able to share their resources between many users. The connection between computers can be done by guided or unguided media i.e. wired and wireless network. The guided media can be connected via cabling most commonly the Ethernet cable. The unguided media can be connected via radio waves. Connected computers or nodes can be shearing their resources, like access to the internet, printers, file servers, and others. A network is a multipurpose connection, which allows a single node to do more.
\end{abstract}

\section{Traffic Engineering}

Traffic Engineering is a technique for advancing the execution of an information transfers system by powerfully breaking down, foreseeing and controlling the conduct of information transmitted over that network. Traffic engineering is also known as tele-traffic engineering and traffic management.

\section{Network Optimization}

Evolving and expanding a network to meet ever-changing demands from consumers and business users is a challenge. The greatest popular impartial is minimizing highest utilization in a normal working situation. One common strategy is reducing maximum operation under de essential failure situations. Also additional objectives occur like minimizing proliferation delay, etc.

\section{Shortest Path routing}

Shortest path routing is the process of finding paths through a network which has minimum of distance to the other cost metric. Here metric is the distance between all routers with each other. In shortest path routing, the topology communications network is represented using a directed weighted graph. The nodes in the graph represent switching elements and the directed arcs in the graph represent communication links between switching elements. Each arc has a weight that represents the cast of sending a packet between two nodes in a particular direction. This cost is generally a positive value that can inculcates such factors as delay, throughput, error rate, monetary cost etc. A path between two nodes may go through several intermediary nodes and arc. The objective in shortest path routing is to find a path between two nodes that has the smallest total cost, where the total cost of a path is the sum of the arc costs in that path. 


\section{Open Shortest Path First}

OSPF was developed as a replacement for the distance vector routing protocol Routing Information Protocol (RIP). It operates as a classless routing protocol that uses the concept of areas for network scalability. RFC 2328 defines the OSPF metric as an arbitrary value called cost. The Cisco IOS uses bandwidth as the OSPF cost metric. OSPF's major advantages over RIP are its fast convergence and its scalability to much larger network implementations. OSPF has a default administrative distance of 110 . As a classless routing protocol, it does not use a Transport layer protocol, as OSPF packets are sent directly over IP.

\section{PROBLEM IDENTIFICATION}

One of the very important practical problems has been the finding of the shortest path between two nodes. These nodes can be as big as countries or as small as atoms. The problem of finding the shortest path between all pairs of vertices on a graph is similar to making a table of all of the distances between all pairs of nodes.

\section{METHODS USED}

\section{Formulation of Entropy Optimization}

Entropy optimization principles are developed to establish some inference criteria for predicting probabilities based on incomplete information. The maximum entropy principle claims: "In making inference on the basis of partial information we must use that probability distribution which has maximum entropy subject to whatever is known. This is the only unbiased assignment we can make".

\section{Shortest Path Betweenness Centrality [1]:}

Let $\sigma_{\mathbf{m}, \mathbf{m}}$ represent the total number of shortest paths between every pair of source-destination nodes $(s, t)$ : $s$ and $t \epsilon V$. Let $\sigma_{\mathbf{n}, \mathbf{E}}(i)$ represent the number of geodesics between every pair of nodes $(\mathrm{s}, \mathrm{t})$ that pass through a link $(i) \in \mathrm{E}$. The Shortest Path Betweenness Centrality (SPBC) of a link $(i)$ is defined as

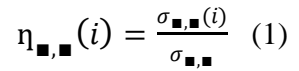

\section{Network Centrality [1]:}

The network centrality or the entropy of SPBC is defined by

$$
\begin{aligned}
& H\left(\Delta_{G}\right)=-\sum_{i \in E} p(i) \log p(i) \\
& \text { Where, } \\
& p(i)=\frac{\eta_{\mathbf{\bullet , \mathbf { }}}(i)}{\sum_{(x, y) \in E} \eta_{\mathbf{\bullet , \mathbf { E }}}(x, y)} \\
& \text { (3) } \\
& \text { for every }(i) \in E
\end{aligned}
$$

$\Delta_{G}$ represents the random variable associated with the probability distribution formed from equation (3). The relative entropy is defined as

$$
h_{G}=\frac{H\left(\Delta_{G}\right)}{\log (\|E\|)}, 0 \leq h_{G} \leq 1
$$

Mathematically, it is stated as the following optimization problem (E1):

$$
\begin{aligned}
& \max H\left(\Delta_{G}\right):=-\sum_{i=1}^{n} p_{i} \ln \mathbf{p}_{i} \\
& \text { s.t. } \sum_{i=1}^{n} \mathbf{p}_{i} \mathbf{f}_{i j}(x)=E\left[\mathbf{f}_{j}\right], j=1,2, \ldots, m
\end{aligned}
$$

(5)

$\sum_{i=1}^{n} p_{i}=1, p_{i} \geq 0, i=1,2, \ldots, n$ where the vector $\mathrm{p}$ stands for the probability to be assigned, $E\left[f_{j}\right]$ denote the $j^{\text {th }}$ moment known from some probabilistic experiments and $\mathrm{H}\left(\Delta_{\mathbf{G}}\right)$ is the Shannon entropy measure.

It can be easily verified that the problem (E1) is a convex programming and has an unconstrained dual program in from

$$
\min _{\mu} D(\mu):=\ln \left\{\sum_{i=1}^{n} \exp \left(\sum_{j=1}^{m} \mu_{j} f_{j i}\right)\right\}-\sum_{j=1}^{m} \mu_{j} E\left[f_{j}\right]
$$

Where $\mu$ is a vector of Log range multiplier, if one has a prior probability $\mathrm{q}=\left(\mathrm{q}_{1}, \mathrm{q}_{2}, \mathrm{q}_{3}, \ldots \ldots ., \mathrm{q}_{\mathrm{n}}\right)$, in addition to the moment constraints in (E1), the probability p should be assigned based on the minimum cross-entropy principle. Mathematically, it leads to the following entropy optimization problem (E2):

$$
\begin{aligned}
& \min D(\mathbf{p}, \mathbf{q}):=\sum_{i=1}^{n} \mathbf{p}_{i} \ln \left(\mathbf{p}_{\mathrm{i}} / \mathbf{q}_{\mathrm{i}}\right) \\
& \text { s.t. } \sum_{\mathrm{i}=1}^{\mathrm{n}} \mathbf{p}_{\mathrm{i}} \mathbf{f}_{\mathrm{ji}}(\mathbf{x})=\mathrm{E}\left[\mathbf{f}_{\mathrm{j}}\right], \mathrm{j}=1,2, \ldots \mathrm{m} \\
& \qquad \sum_{\mathrm{i}=1}^{\mathrm{n}} \mathbf{p}_{\mathrm{i}}=1, \mathbf{p}_{\mathrm{i}} \geq \mathbf{0}, \mathbf{i}=1,2, \ldots, \mathrm{n}
\end{aligned}
$$

Where $\mathrm{D}(\mathrm{p}, \mathrm{q})$ stands for the Kullback-Leibler's cross-entropy or relative entropy.

\section{Dijkstra's Algorithm:}

Dijkstra's algorithm is also known as single source shortest path algorithm. It calculates the shortest path from the source to each of the unvisited vertices in the graph. It used the method of increasing node by node to get a shortest path tree which makes the starting point as its root.

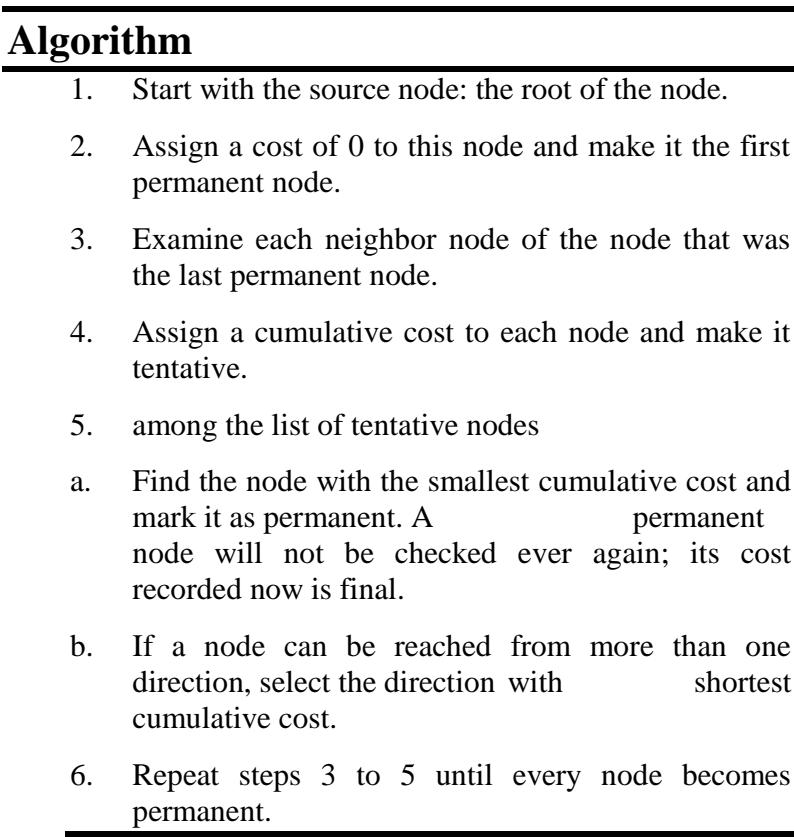

\section{Floyd Warshall Algorithm:}

Floyd-Warshall algorithm is an algorithm for finding most brief ways in a weighted chart with positive or negative edge weights however with no negative cycles. A solitary execution of the calculation will discover the lengths of the briefest ways between all sets of vertices; however it doesn't return subtle elements of the ways themselves. Variants of the calculation can likewise be utilized for finding the transitive conclusion of a connection $\mathrm{R}$, or in amplest ways between all sets of vertices in a weighted chart. 


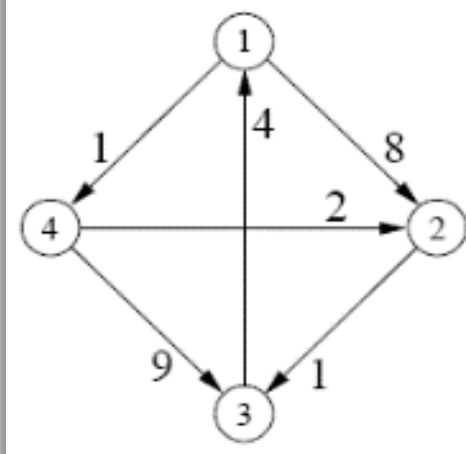

(a)
$d_{3,2}^{(0)}=I N F$ (no path)

$d_{3,2}^{(1)}=12(3,1,2)$

$d_{3,2}^{(2)}=12(3,1,2)$

$d_{3,2}^{(3)}=12(3,1,2)$

$d_{3,2}^{(4)}=7 \quad(3,1,4,2)$

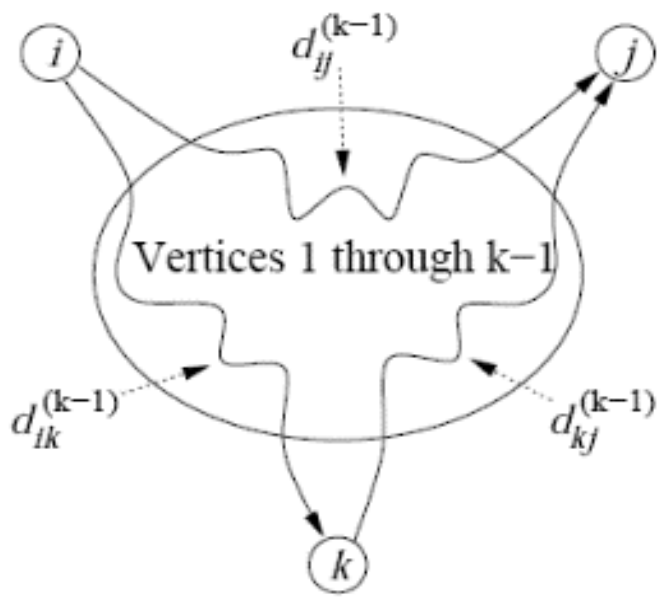

Figure 1: Floyd-Warshall Algorithm

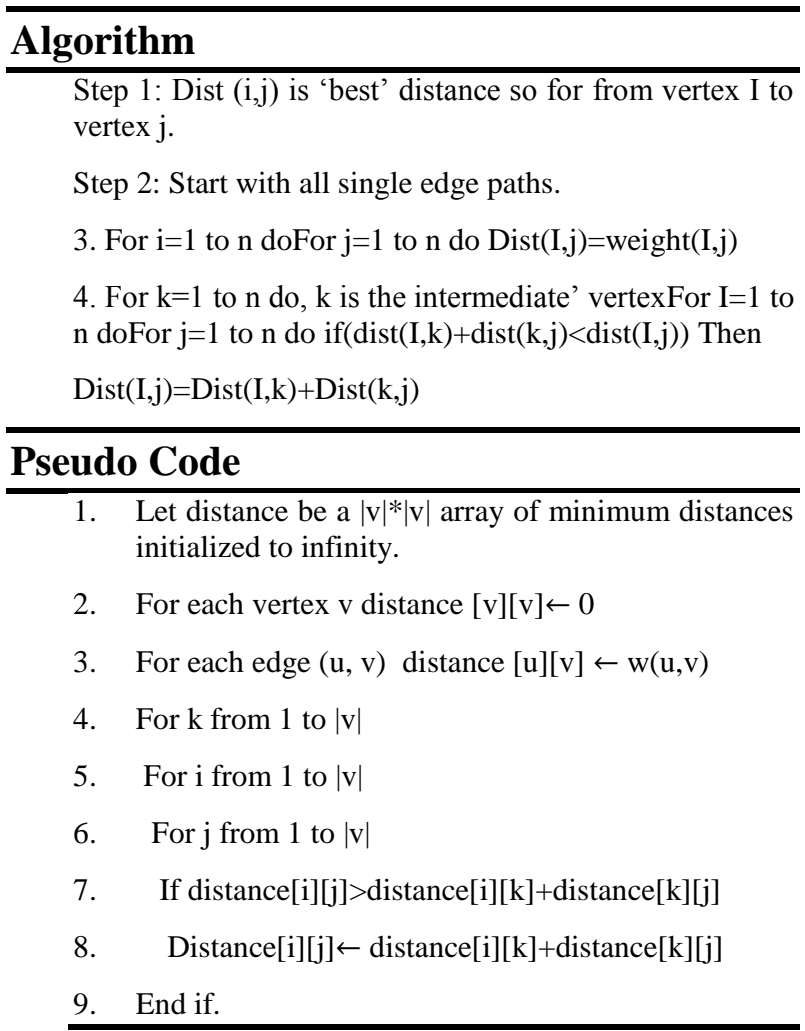

\section{The proposed Algorithm (HDFWA)}

Proposed algorithm Hybrid Dijkstra's-Floyd Warshall Algorithm (HDFWA) can also be used for finding shortest path i.e. lowest cost from origin node to destination node in a network graph. The input of the algorithm consists of distances directed between nodes as $r$ and a source data from origin and destination paths as $s, d$ in the network graph $\mathrm{G}$. If the distance between two nodes can be defined as path cost, the total cost of a network graph is the sum of all route distances between nodes in a graph.

\section{Algorithm \\ Step 1: Node weight computation for all nodes //for each node \\ and edge pick the costs in $\mathrm{W}(\mathrm{k})$.For $\mathrm{i}=$ =start to last edges \\ For $\mathrm{j}=$ first to last edges $/ / \mathrm{j}$ is set to the destination node if $(\mathrm{G}(2, \mathrm{j})=\mathrm{i}) / / \mathrm{k}$ is set to the source node $\mathrm{W}(\mathrm{k})=\mathrm{W}(\mathrm{i})$ $+\mathrm{G}(3$ \\ ,j);}

Step 2: Shortest Path computation For $i=$ first to last edges while (the origin (k)is the same in graph, $\mathrm{G}$ )

$$
\begin{aligned}
& \text { if }(\mathrm{G}(3, \mathrm{i})=\mathrm{W}(\mathrm{k})-\mathrm{W}(\mathrm{j})) \\
& \mathrm{P}(\mathrm{k})=\mathrm{G}(2, \mathrm{i}) ; \text { elsei }=\mathrm{i}+1 ; \\
& \mathrm{k}=\mathrm{G}(1, \mathrm{i}) \text {; End }
\end{aligned}
$$

\section{RESULT}

Experimental results of the proposed algorithm for solving shortest path routing are shown. These results show the significant improvement of the Dijkstra's-Floyd Warshall. There is a combination of several parameters which affect the algorithm performance. In this work the effect of a combination of these parameters in the algorithm will be analyzed. The values according to numerous performances of the program are considered to achieve near-optimal solution to increase optimum. The performance of Floyd WarshallDijkstra's is affected by various factors such as parameters, Floyd operators and strategies. The traditional approach with random initial population is efficient however the whole initial population may contain feasible solutions, which causes Floyd Warshall-Dijkstra's take less time to converge. The Floyd Warshall-Dijkstra's is modified in various ways to achieve faster and better convergence and it was particularly recognized by the researchers that initial path greatly affects the performance of Floyd Warshall-Dijkstra's (Proposed work). This study proposes modified Floyd WarshallDijkstra's with sorted initial stage and applies it to solving shortest path routing (SPR). New approach will allow reducing the size of the initial problem while preserving a better fit and this leading to faster and better convergence. Floyd Warshall-Dijkstra's is based on theory of NP-hard problem and new approach sorts out initial. It gives grounds 
to assume that a better solution can be obtained and in a shorter time. Moreover, new approach proposes that Floyd Warshall-Dijkstra's algorithm has less probability of being stuck in the local optimum and rather produces a result close to global optimum.

In figure 2, we get max entropy in proposed work which is '7'

In figure 3, we show the path $\{1,6,40,4,69,2,15,50\}$ here first node is source node ' 1 ' and last node is destination node ' 50 ' and other are traverse path ' $6,40,4,69,2,15$ '.

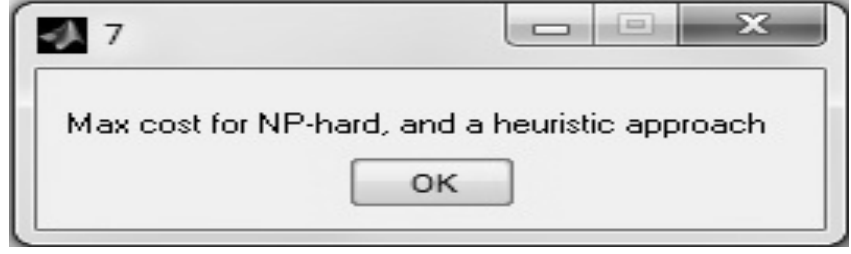

Figure 2: Maximum Entropy

\begin{tabular}{llllllll|lll|l}
1 & 6 & 40 & 4 & 69 & 2 & 15 & 50 & $\square$ & $\square$ & $\Sigma$ \\
\hline
\end{tabular}

Optimized Path for NP-hard, and heuristic approach

OK

Figure 3: Travels Path

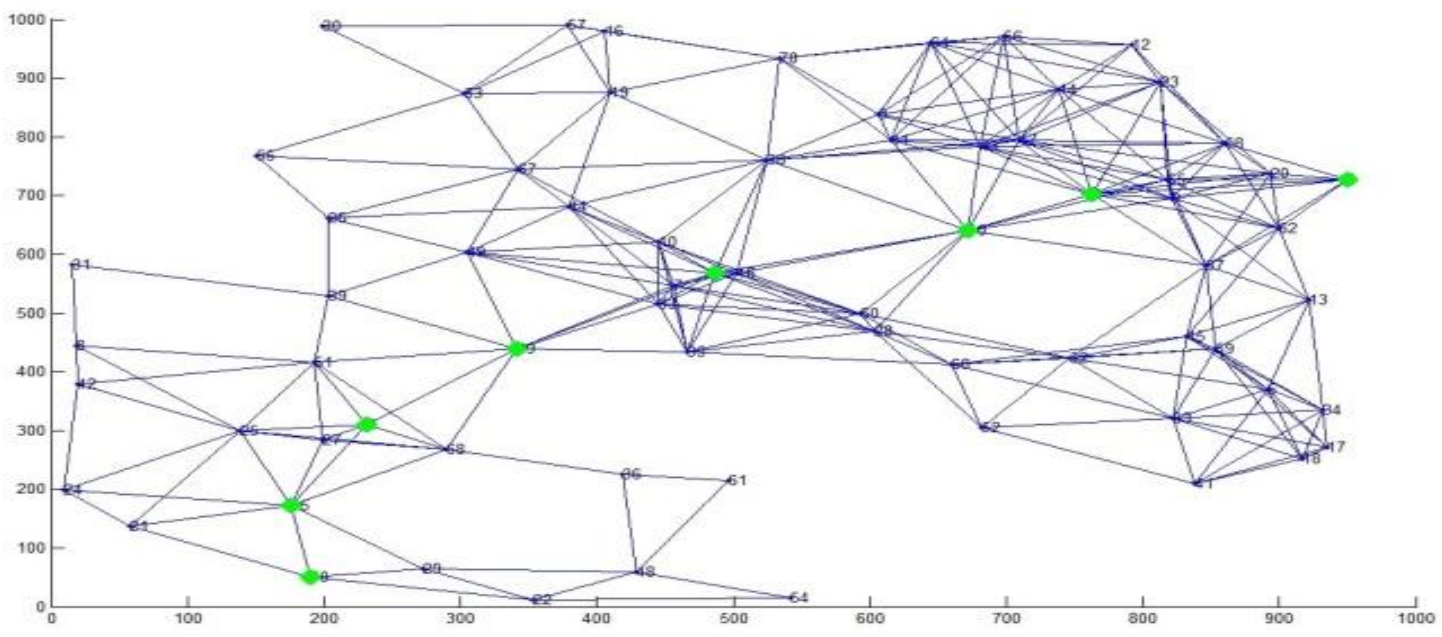

Figure 4: Network Topology

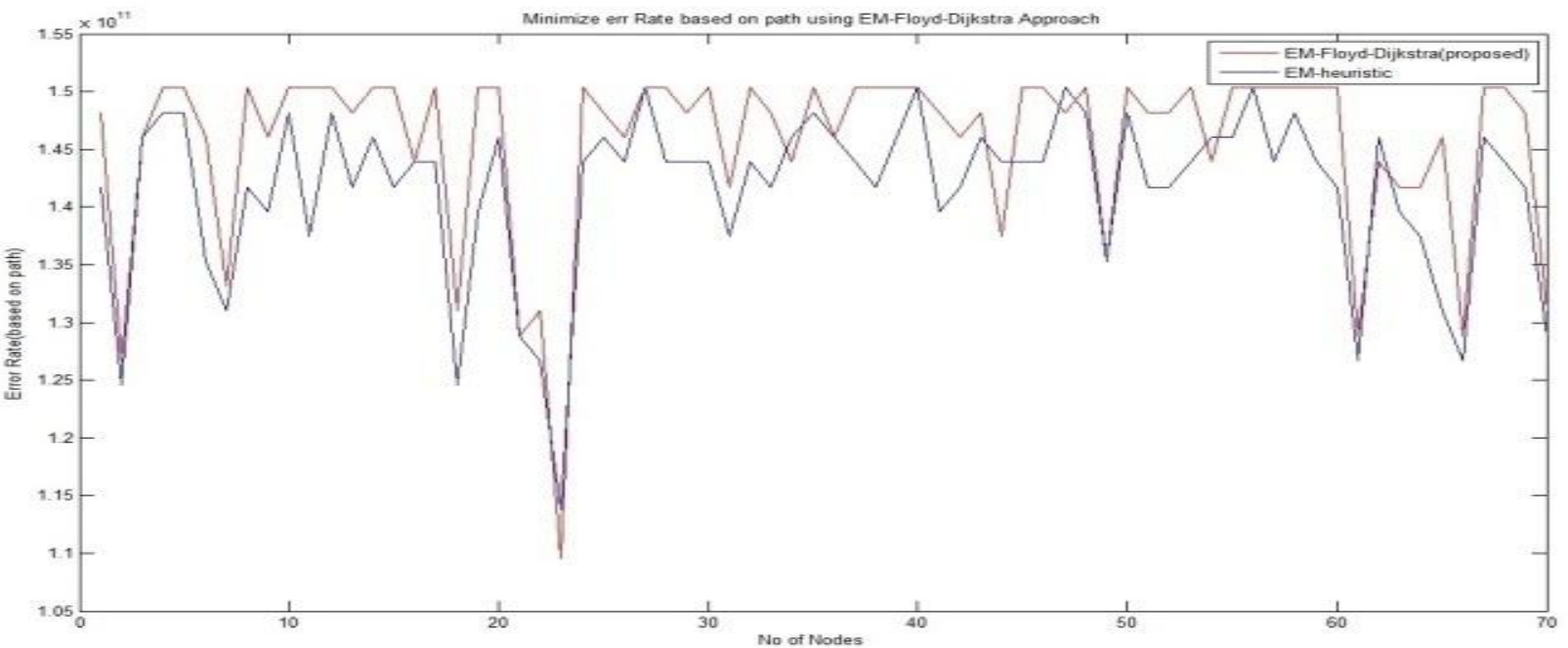

Figure 5: Error rate of EM-Dijkstra's-Floyd and EM-Heuristic

In figure 5, we observed that the EM-Floyd Dijkstra's Algorithm is batter then Error rate of EM-heuristic. Here, we get the minimum error rate at entropy 7 when number of nodes are 70 , where source node and destination node are 1 and 50 which is $1.1 \times 10^{11}$.

\section{CONCLUSION}

After studied these two algorithms I conclude that HDFWA is very faster than existing algorithm. That's why I have chosen Dijkstra's-Floyd Warshall algorithm for finding shortest path in Network. It is also work better and faster in very larger network. Dijkstra-Floyd Warshall algorithm calculates the shortest distance from node to node that is suitable for systems that have a large number of nodes. For larger path, there are many of nodes, which Dijkstra's algorithm requires a 
lot of time and cost. However, in term of shortest path, Floyd Warshall-Dijkstra's give better results than EM-heuristic algorithm but this improvement just is approximately 10 to 15 percent while it has less important versus of the processing time, especially in the wide area. Floyd Warshall-Dijkstra's algorithm is a heuristic algorithm to search for a new route uses heuristic information. The lower nodes and therefore less time are required. For improve the effectiveness of method Floyd Warshall-Dijkstra's is used from the restriction direction, so with it eliminated nodes that are moving in the opposite direction and further the scrolling speed rises.

In future studies, the angular size of the proposed method and floating-point values for the various routes can be determined.

\section{REFERENCES}

[1] Vanniarajan Chellappan, Krishna M. Sivalingam, and Kamala Krithivasan. "An Entropy Maximization Problem in Shortest Path Routing Networks." Local \& Metropolitan Area Networks (LANMAN), 2014 IEEE 20th International Workshop on. IEEE, 2014

[2] LI Ji, WANG Bing-Hong, WANG Wen-Xu, ZHOU Tao, "Network entropy based on topology configuration and its computation to random networks." Chinese Physics Letters 25.11 (2008): 4177.

[3] J. N. Kapur and H. K. Kesvan, "Entropy optimization principles and their applications. Springer Netherlands, 1992.
[4] Marco Frittelli, "The minimal entropy martingale measure and the valuation problem in incomplete markets." Mathematical finance 10.1 (2000): 39-52.

[5] Ginestra Bianconi, Entropy of network ensembles." Physical Review E79.3 (2009): 036114.

[6] Brian P. Tighe, Adrianne R.T. van Eerd, and Thijs J. H. Vlugt, "Entropy maximization in the force network ensemble for granular solids." Physical review letters 100.23 (2008): 238001

[7] Naonori Ueda and Ryohei Nakano, "Deterministic annealing EM algorithm."Neural Networks 11.2 (1998): 271-282.

[8] Maximilien Gadouleau and Soren Riis, "Graphtheoretical constructions for graph entropy and network coding based communications." Information Theory, IEEE Transactions on 57.10 (2011): 6703-6717.

[9] S. Mart'ınez, F. Nicol'as, F. Pennini, and A. Plastino, "Tsallis' entropy maximization procedure revisited."Physica A: Statistical Mechanics and its Applications 286.3 (2000): 489-502.

[10] R.L.C. Vink and G.T. Barkema, "Configurational entropy of network-forming materials." Physical review letters 89.7 (2002): 076405.

[11] Babak Hassibi, Sormeh Shadbakht "Normalized entropy vectors, network information theory and convex optimization." Information Theory for Wireless Networks, 2007 IEEE Information Theory Workshop on. IEEE, 2007. 